\title{
Low-Energy Theorems in Two-Nucleon Scattering
}

\author{
A. A. Filin, ${ }^{* a}$ V. Baru ${ }^{b c d}$ and E. Epelbaum ${ }^{a}$ \\ ${ }^{a}$ Ruhr University Bochum, Faculty of Physics and Astronomy, Institute for Theoretical Physics II, \\ D-44780 Bochum, Germany \\ ${ }^{b}$ Helmholtz-Institut für Strahlen- und Kernphysik and Bethe Center for Theoretical Physics, \\ Universität Bonn, D-53115 Bonn, Germany \\ ${ }^{c}$ Institute for Theoretical and Experimental Physics, B. Cheremushkinskaya 25, 117218 Moscow, \\ Russia \\ ${ }^{d}$ P.N. Lebedev Physical Institute of the Russian Academy of Sciences, 119991, Leninskiy \\ Prospect 53, Moscow, \\ E-mail: arseniy.filinarub.de, vadimbetp2.rub.de, \\ evgeny.epelbaumerub.de
}

\begin{abstract}
One-pion exchange defines the longest-range part of the NN interaction, controls the nearthreshold energy dependence of the scattering amplitude and imposes correlations between the coefficients in the effective range expansion. These correlations may be regarded as low-energy theorems. We discuss the low-energy theorems for unphysical pion masses and consider various applications to test recent lattice QCD results. In particular, we demonstrate that the binding energies of the deuteron and dineutron obtained by the NPLQCD Collaboration at a pion mass of $M_{\pi}=450 \mathrm{MeV}$ are inconsistent with the low-energy behavior of the corresponding phase shifts within the quoted uncertainties. Using the binding energies of the deuteron and dineutron as input, we employ the low-energy theorems to predict the phase shifts and extract the scattering length and the effective range in the ${ }^{3} S_{1}$ and ${ }^{1} S_{0}$ channels.
\end{abstract}

The 9th International workshop on Chiral Dynamics 17-21 September 2018

Durham, NC, USA

\footnotetext{
* Speaker.
} 


\section{Introduction}

Lattice-QCD allows one to calculate nuclear observables from first principles and to study their light-quark-mass-dependence (or, equivalently, pion-mass dependence). Recent advances in lattice-QCD allow fully dynamical calculations for unphysical pion masses as low as $M_{\pi} \simeq 300$ $400 \mathrm{MeV}$ in NN systems $[1,2,3]$ and even for $M_{\pi} \sim M_{\pi}^{\text {phys }}$ for baryon-baryon interactions with non-zero strangeness $[4,5]$. In addition to binding energies also scattering phase shifts became accessible on the lattice. For example, the NPLQCD collaboration analyzed NN scattering in ${ }^{1} S_{0}$ and ${ }^{3} S_{1}$ channels at $M_{\pi} \simeq 450$ and $800 \mathrm{MeV}[3,6,7,8]$ and the CalLat collaboration presented higher partial waves at $M_{\pi} \simeq 800 \mathrm{MeV}[9,10]$. In spite of large progress in lattice simulations, lattice-QCD results for the binding energies of the deuteron $\left({ }^{3} S_{1}\right)$ and dineutron $\left({ }^{1} S_{0}\right)$ for largerthan-physical pion masses are still puzzling, because there is no agreement between different calculations. Many groups report that both the deuteron and dineutron become more bound with increased pion mass $[1,2,3,7,11]$. On the other hand, the HAL QCD collaboration (which uses a different approach from the references above) finds no bound states in the ${ }^{3} S_{1}$ and ${ }^{1} S_{0}$ channels for the pion-mass range from 469 to $1171 \mathrm{MeV}$ [12]. To connect lattice results at pion masses slightly larger than the physical one with experimental data one can use effective field theory (EFT) $[13,14,15,16,17,18,19,20,21]$. It is remarkable that many EFT calculations suggest less attraction in ${ }^{3} S_{1}$ and ${ }^{1} S_{0}$ channels for higher-than-physical pion masses [13, 16, 15, 21, 20]. These calculations, however, use extra assumptions (such as naturalness and resonance saturation estimates) about $M_{\pi}$-dependent four-nucleon contact interactions.

Alternatively, to study the properties of NN scattering at a given pion mass and to check selfconsistency of lattice-QCD results, a method of low-energy theorems (LETs) can be employed [22] (see also Refs. [23, 24, 25, 26, 27, 28] for earlier studies). Specifically, using knowledge about the long-range part of the interaction and analytic properties of the scattering amplitude one can predict correlations (and, in some cases, relations) between observables. LETs were tested in Ref. [22] at the physical pion mass to predict effective range parameters for $\mathrm{NN}$ scattering in ${ }^{3} S_{1}$ and ${ }^{1} S_{0}$ channels. In Ref. [22], LETs were also generalized to unphysical pion masses and correlations between effective range expansion (ERE) parameters were derived.

Recently, the NPLQCD collaboration performed a lattice calculation of the binding energies and phase shifts in ${ }^{3} S_{1}$ and ${ }^{1} S_{0} \mathrm{NN}$ scattering at $M_{\pi}=450 \mathrm{MeV}$ [3]. In this contribution we follow Ref. [29] and compare these results with predictions of LETs. Specifically we use the binding energies obtained by NPLQCD as input for LETs to predict phase shifts in ${ }^{3} S_{1}$ and ${ }^{1} S_{0}$ channels and then to compare them to the phase shifts calculated on the lattice. We observe that the lattice phase shifts calculated at two lowest energy points are inconsistent with the lattice binding energies within quoted uncertainties. We also employ LETs to calculate the values of the scattering length and effective range, based on the lattice binding energy.

\section{Formalism of low-energy theorems}

In this section we follow Refs. [22, 29] to briefly summarize the main points of the LETs formalism. We consider low-energy NN scattering using a potential which can be separated into the long- and short-range parts, where the long-range part corresponds to the one-pion exchange 
A. A. Filin,

potential (OPEP). The short-distance part is unknown, but assumed to be sufficiently short-ranged such that its impact can be parameterized just by several parameters. ${ }^{1}$ At leading order (LO) we assume that the effect of the short-range potential can be parameterized by a single parameter (for a given partial wave). At next-to-leading order (NLO) we parameterize it by two parameters and at higher orders more parameters should be introduced. After solving the nonperturbative scattering problem for the sum of the short- and long-range potentials we obtain the scattering observables and binding energies. Since the long-range interaction is fixed, all observables turn out to be functions of several unknown parameters which parameterize effects of the short-range interaction. This implies that all scattering observables are correlated by long-range interactions. These correlations can be interpreted as LETs. Specifically, at LO all observables are functions of a single parameter. Thus, we can choose one observable (for example, the scattering length) and re-express all remaining observables in terms of this single observable.

To implement LETs, we use the EFT formulation discussed in Refs. [22, 29]. The scattering amplitude $T$ in this formulation is calculated as a solution of the Lippmann-Schwinger-type integral equation introduced originally by Kadyshevsky [31]:

$$
T\left(\vec{p}, \vec{p}^{\prime}, k\right)=V\left(\vec{p}, \vec{p}^{\prime}\right)+\int d^{3} q V(\vec{p}, \vec{q}) G(k, q) T\left(\vec{q}, \vec{p}^{\prime}, k\right),
$$

where $V=V_{\text {Long }}+V_{\text {Short }}$, the free Green's function is defined as

$$
G(k, q)=\frac{m_{N}^{2}}{2(2 \pi)^{3}} \frac{1}{\left(\vec{q}^{2}+m_{N}^{2}\right)\left(E_{k}-\sqrt{\vec{q}^{2}+m_{N}^{2}}+i \varepsilon\right)},
$$

$\vec{p}\left(\vec{p}^{\prime}\right)$ denotes the incoming (outgoing) three-momentum of the nucleon in the cms and $E_{k}=$ $\sqrt{\vec{k}^{2}+m_{N}^{2}}$ with $m_{N}$ standing for the nucleon mass and $\vec{k}$ being the corresponding three-momentum. The long-range part of the interaction potential is defined by one-pion-exchange potential (OPEP):

$$
V_{\text {Long }}\left(\vec{p}, \vec{p}^{\prime}\right)=-\frac{g_{A}^{2}}{4 F_{\pi}^{2}} \frac{\vec{\sigma}_{1} \cdot\left(\vec{p}-\vec{p}^{\prime}\right) \vec{\sigma}_{2} \cdot\left(\vec{p}-\vec{p}^{\prime}\right)}{\left(\vec{p}-\vec{p}^{\prime}\right)^{2}+M_{\pi}^{2}} \tau_{1} \cdot \tau_{2}
$$

where $\vec{\sigma}_{i}\left(\boldsymbol{\tau}_{i}\right)$ denote the spin (isospin) Pauli matrices of the nucleon $i, g_{A}$ is axial vector coupling of the nucleon and $F_{\pi}$ is the pion decay constant. The short-range interaction at $\mathrm{LO}$ is parameterized by a derivative-less (momentum-independent, zero-range) contact interaction $V_{\mathrm{Short}}^{\mathrm{LO}}=C_{0}$. We use the Kadyshevsky equation (2.1)-(2.2) instead of the standard Lippmann-Schwinger equation (LSE), because for the potentials we are considering, this equation is proven to be exactly renormalizable [32]. Therefore one can set the cutoff in Eq. (2.1) to infinity to avoid practical problems related with the finite cutoff artifacts. In principle, one can also use the standard LSE with a finite cutoff to implement the LETs, if the finite-cutoff effects are properly controlled.

At NLO, the finite-range effects of the short range interaction are taken into account. To introduce NLO contact interactions and still preserve explicit renormalizability we use the resonance

\footnotetext{
${ }^{1}$ Effects of the short range potential on low-energy scattering can be parameterized in various ways. For example, one can parameterize them by the scattering length, effective range and shape parameters of the short-range part of the potential [30]. In the EFT formulation, the effects of short range interactions are encoded in low-energy constants.
} 
saturation method [22]. The NLO correction to the potential is introduced as a heavy-meson exchange potential:

$$
V_{\text {Short }}^{\mathrm{NLO}}\left(\vec{p}, \vec{p}^{\prime}\right)=\beta \frac{\vec{\sigma}_{1} \cdot\left(\vec{p}-\vec{p}^{\prime}\right) \vec{\sigma}_{2} \cdot\left(\vec{p}-\vec{p}^{\prime}\right)}{\left(\vec{p}-\vec{p}^{\prime}\right)^{2}+M^{2}},
$$

where the heavy-meson mass $M$ is chosen to be $700 \mathrm{MeV}$ and the strength $\beta$ parameterizes the finite-range effects of the short range interaction. For the physical pion mass the value of $\beta$ is fitted to reproduce the empirical value of the effective range in the ${ }^{1} S_{0}$ and the ${ }^{3} S_{1}$ channels (separately for each partial wave). The functional form of the NLO term (i.e. spin-angular structure, the value of heavy-meson mass) is not important as long as this term remains of a short range.

To get quantitative predictions based on LETs, we solve the coupled-channel ${ }^{2}$ integral equation (2.1) numerically in the partial wave basis and get the scattering amplitude $T$ as a function of the contact terms. For an uncoupled channel with zero orbital angular momentum $\left({ }^{1} S_{0}\right)$, the on-shell scattering amplitude $T(k) \equiv T(k, k, k)$ can be expressed in terms of the so-called effective range function $F(k) \equiv k \cot \delta(k)$ via

$$
T(k)=-\frac{16 \pi^{2}}{m_{N}} \frac{1}{F(k)-i k} .
$$

The effective range function is easier to parameterize than the scattering amplitude, because it does not have the kinematic unitarity cut and is a real meromorphic function of $k^{2}$ in the vicinity of $k=0[33,34]$. It can be expanded into Taylor-series around $k=0$ leading to the effective range expansion:

$$
F(k)=k \cot \delta(k)=-\frac{1}{a}+\frac{1}{2} r k^{2}+v_{2} k^{4}+v_{3} k^{6}+v_{4} k^{8}+\ldots,
$$

where $a$ is the scattering length, $r$ is the effective range, and $v_{i}$ are the so-called shape parameters. For the coupled-channel ${ }^{3} S_{1}-{ }^{3} D_{1}$ problem the above formalism is generalized to include the ${ }^{3} D_{1}$ phase shift and ${ }^{3} S_{1}-{ }^{3} D_{1}$ mixing angle.

To implement the LETs at LO, a single observable is used as input to fix the short range parameter, and other observables are then predicted. Alternatively, one can plot scattering observables as functions of the input. For example, the ERE parameters as functions of the scattering length are calculated in Ref. [22]. For NLO LETs one has to fix one more parameter. At the physical pion mass we use the empirical values of the effective range in ${ }^{1} S_{0}$ and ${ }^{3} S_{1}$ channels to fix the NLO parameters.

Generalization of LETs to unphysical pion masses is performed in a straightforward way [22]. The largest effect comes from the variation of the pion mass in the propagator of OPEP. Additional effects come from the $M_{\pi}$-dependence of $F_{\pi}, m_{N}$, and $g_{A}$. We use the fits of lattice data performed in Ref. [22] to determine $F_{\pi}, m_{N}$, and $g_{A}$ at unphysical pion masses.

The short-range parameters should be fixed from observables at a given unphysical pion mass. For LO LETs the single short-range parameter can be determined, for example, from the scattering length or from the binding energy at a given unphysical pion mass. Unlike chiral extrapolations, no assumptions are made about $M_{\pi}$-dependence of the short range parameter $C_{0}$.

At NLO there is one more parameter, which parameterizes the short-range effects. There are several strategies which can be used to determine its value or a range of values. If there is enough

\footnotetext{
${ }^{2}$ We solve coupled-channel equation for ${ }^{3} S_{1}-{ }^{3} D_{1}$ case, and single channel equation for ${ }^{1} S_{0}$ case.
} 
lattice data, it can be fixed from some observable at the unphysical pion mass. In practice, we assume that the parameter $\beta$ does not change dramatically compared to its value at the physical pion mass. Note that unnaturally large values of $\beta$ will increase the finite-range effects of the short range potential and thus violate our main assumption that long-range effects are dominated by OPEP. To quantify the range of $\beta$ values, we introduce dimensionless parameter $\delta \beta$, which defines the natural range of $\beta$ for pion masses larger than the physical one. Formally, we restrict the $M_{\pi}^{2}$-dependence of $\beta$ by the following inequality:

$$
1-\delta \beta\left|\frac{M_{\pi}^{2}-\left(M_{\pi}^{\text {phys }}\right)^{2}}{\Delta M_{\pi}^{2}}\right| \leq \frac{\beta\left(M_{\pi}\right)}{\beta\left(M_{\pi}^{\text {phys }}\right)} \leq 1+\delta \beta\left|\frac{M_{\pi}^{2}-\left(M_{\pi}^{\text {phys }}\right)^{2}}{\Delta M_{\pi}^{2}}\right|,
$$

with $\Delta M_{\pi}^{2} \equiv\left(M_{\pi}^{\text {ref }}\right)^{2}-\left(M_{\pi}^{\text {phys }}\right)^{2}, M_{\pi}^{\text {ref }}=500 \mathrm{MeV}$, and $\beta\left(M_{\pi}^{\text {phys }}\right)$ fixed at the physical pion mass. For our central ${ }^{3} S_{1}$ results we use $\delta \beta=0.5$, which means that $\beta$ can change by $50 \%$ when going from the physical value of $M_{\pi}$ to $M_{\pi}=500 \mathrm{MeV}$. This choice of $\delta \beta$ is motivated by the fact that it would cover the known $M_{\pi}$-dependence of $g_{A}, F_{\pi}$ and $m_{N}$ if the same procedure is applied to these quantities. In the next section, we will also give results corresponding to the more conservative choice of $\delta \beta=1.0$. For calculations in the ${ }^{1} S_{0}$ channel we use $\delta \beta=1.0$, due to a larger role of short-range forces in that channel. We have checked that introducing a reasonable ${ }^{3} M_{\pi}$-dependence in the heavy meson mass does not increase the uncertainty, because its effect is simulated by the variation of $\beta$.

\section{Analysis of NPLQCD results at $M_{\pi}=450 \mathrm{MeV}$}

The NPLQCD collaboration in Ref. [3] reported their results for lattice calculations of the deuteron and dineutron binding energies and the ${ }^{1} S_{0}$ and ${ }^{3} S_{1}$ phase shifts at the pion mass $M_{\pi} \simeq 450$ $\mathrm{MeV}$. The deuteron and dineutron binding energies quoted in Ref. [3] at $M_{\pi} \simeq 450 \mathrm{MeV}$ are

$$
B_{d}=14.4\left(\begin{array}{c}
+3.2 \\
-2.6
\end{array}\right) \mathrm{MeV}, \quad B_{n n}=12.5\left({ }_{-5.0}^{+3.0}\right) \mathrm{MeV},
$$

where the errors come from statistical, systematic, and extrapolation uncertainties combined in quadrature. The phase shifts calculated in Ref. [3] and the corresponding uncertainties are shown as filled black regions in Fig. 1 (left) for ${ }^{3} S_{1}$ and Fig. 2 (left) for ${ }^{1} S_{0}$. Further, the obtained deuteron binding energy and two lowest-energy phase shift data points are used in Ref. [3] to extract the ERE parameters using the effective range approximation

$$
k \cot \delta \simeq-\frac{1}{a}+\frac{1}{2} r k^{2}
$$

The result of this fit is shown by gray bands in Fig. 1 (right) and Fig. 2 (right). The inverse scattering length and the effective range extracted from this fit in Ref. [3] read:

$$
\begin{aligned}
& \left(M_{\pi} a_{\mathrm{NPLQCD}}^{\left({ }^{3} S_{1}\right)}\right)^{-1}=-0.04\left({ }_{-0.10}^{+0.07}\right)\left({ }_{-0.17}^{+0.08}\right), \quad M_{\pi} r_{\mathrm{NPLQCD}}^{\left({ }^{3} S_{1}\right)}=7.8\left({ }_{-1.5}^{+2.2}\right)\left(\begin{array}{c}
+3.5 \\
-1.7
\end{array}\right) \\
& \left.\left.\left(M_{\pi} a_{\mathrm{NPLQCD}}^{(1}\right)_{0}\right)=0.021\left({ }_{-0.036}^{+0.028}\right)\left({ }_{-0.063}^{+0.032}\right), \quad M_{\pi} r_{\mathrm{NPLQCD}}^{(1} S_{0}\right)=6.7\left({ }_{-0.8}^{+1.0}\right)\left({ }_{-1.3}^{+2.0}\right),
\end{aligned}
$$

where the uncertainties in the first and second brackets are statistical and systematic, respectively.

\footnotetext{
${ }^{3}$ Of the same order as $M_{\pi}$-dependence of other masses.
} 

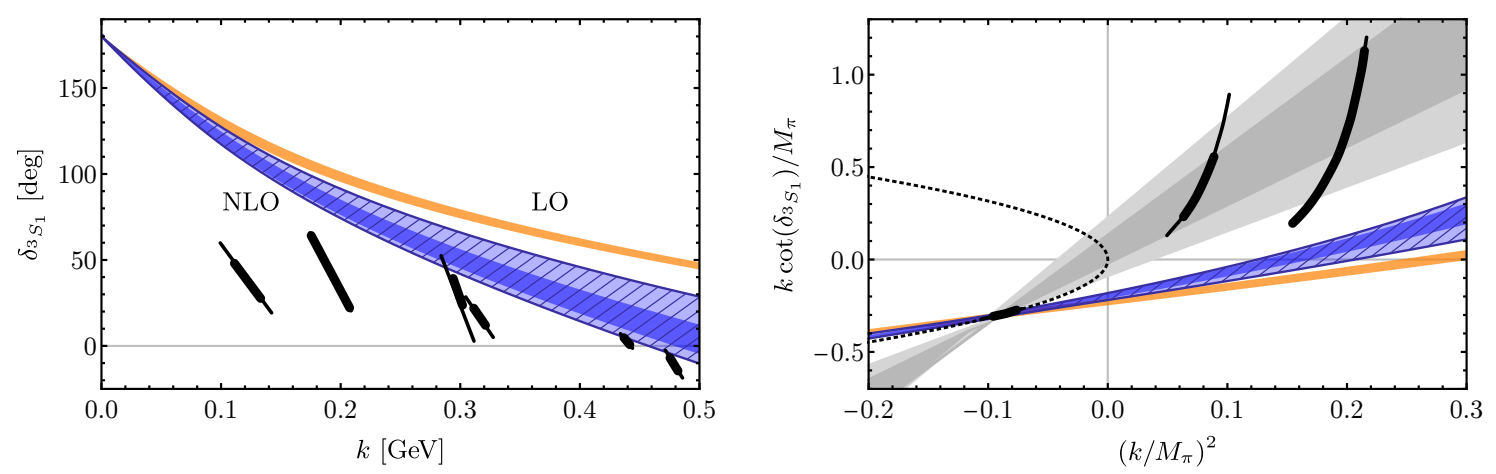

Figure 1: Neutron-proton phase shifts (left panel) and the effective-range function (right panel) in the ${ }^{3} S_{1}$ channel calculated on the lattice at $M_{\pi} \sim 450 \mathrm{MeV}$ [3] (filled black regions) in comparison with the predictions based on the LETs at LO (orange light-shaded bands) and NLO (blue dark-shaded and hatched blue light-shaded bands) using the NPLQCD result for the deuteron binding energy $B_{d}$ as input. The uncertainty at LO shown by the orange bands is entirely given by the uncertainty of $B_{d}$ in Eq. (3.1). The NLO darkshaded (hatched light-shaded) bands correspond to the uncertainty in $B_{d}$ and the theoretical uncertainty of the LETs estimated via the variation of $\beta$ with $\delta \beta=0.5(\delta \beta=1.0)$ combined in quadrature. The gray light- and dark-shaded bands in the right panel depict the fit results of the lattice points of Ref. [3] based on the effective range approximation (3.5). The energy of the bound (virtual) states corresponds to the intersection points of the effective-range function $k \cot \delta^{\left(3 S_{1}\right)}$ and the unitarity term $i k / M_{\pi}= \pm \sqrt{-\left(k / M_{\pi}\right)^{2}}$, shown by the dotted line in the right panel, in the lower (upper) half-plane. The phase shift corresponds to the Blatt-Biedenharn parametrization of the S-matrix [35].

\subsection{Application of LETs to ${ }^{3} S_{1}$ channel}

We now take NPLQCD's value of the deuteron binding energy $B_{d}$ of Eq. (3.1) as input and employ LETs to predict the phase shifts in the ${ }^{3} S_{1}$ channel. At LO, the single parameter in LETs is fixed by the deuteron binding energy, and at NLO an extra parameter is fixed as discussed in Section 2. Our LET-based prediction of the phase shift and the effective range function are shown in Fig. 1 as orange (LO) and blue (NLO) bands. The width of the LO (orange) band corresponds to the uncertainty in the input binding energy of Eq. (3.1) and does not include the theoretical uncertainty of the LETs. The accuracy of the NLO LETs can be estimated by the width of the blue band, which corresponds to the variation of $\beta$ parameter using $\delta \beta=0.5$. This variation is consistent with the shift from LO to NLO LETs. As can be seen from Fig. 1 (left), the NLO LET prediction for ${ }^{3} S_{1}$ phase shifts agrees with lattice results at higher momenta, but disagrees at lower momenta within reported uncertainties. It is also remarkable that the lattice phase shifts tend to $0^{\circ}$ at $k=0$, while phase shifts generated by LETs go to $180^{\circ}$ in agreement with the Levinson's theorem. We have checked that using a much stronger $M_{\pi}$-dependence of $\beta$ than of other coupling constants and masses does not change our conclusions. For illustration, the results of NLO LETs with $\delta \beta=1$ are shown in Fig. 1 as hatched blue band. We observe that even a very conservative estimation of NLO terms cannot remove the disagreement between LETs and lattice data at low energies.

As a next step, we extract the scattering length and effective range corresponding to the phase 

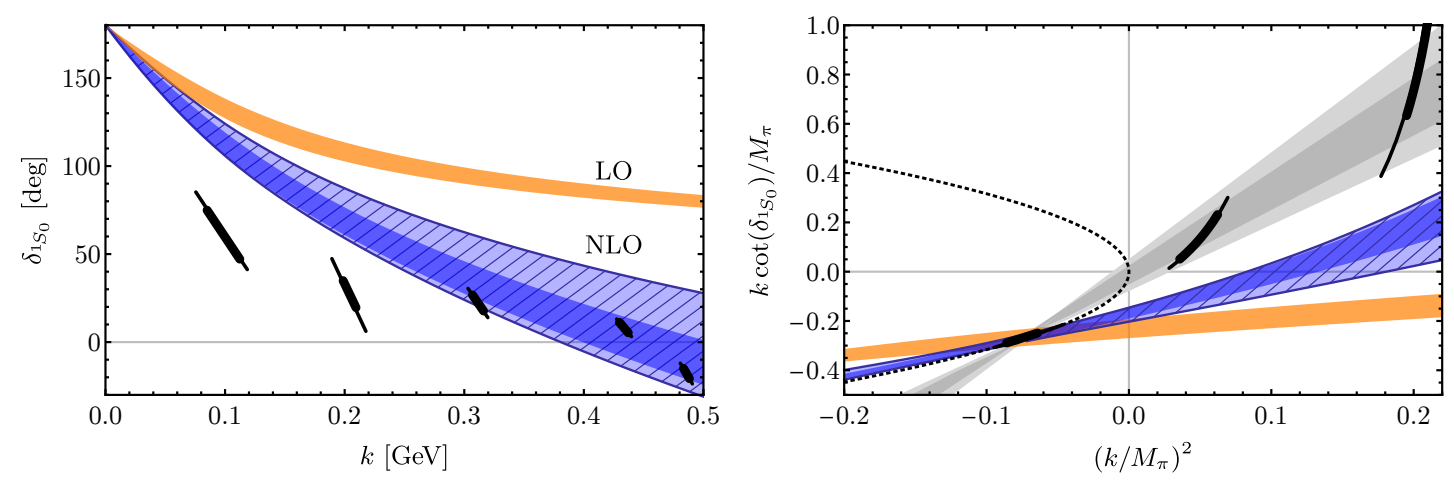

Figure 2: Two-nucleon phase shifts (left panel) and the effective-range function (right panel) in the ${ }^{1} S_{0}$ channel calculated on the lattice at $M_{\pi} \sim 450 \mathrm{MeV}$ [3] in comparison with the predictions based on the LETs at LO and NLO using the NPLQCD result for the dineutron binding energy of Eq. (3.1) as input. For notation see Fig. 1.

shifts generated by LETs. We obtain the following results based on LO and NLO calculations:

$$
\begin{aligned}
& \left(M_{\pi} a_{\mathrm{LET}, \mathrm{LO}}^{\left({ }^{3} S_{1}\right)}\right)^{-1}=0.229\left({ }_{-0.018}^{+0.019}\right), \quad M_{\pi} r_{\mathrm{LET}, \mathrm{LO}}^{\left({ }^{3} S_{1}\right)}=1.62\left({ }_{-0.06}^{+0.06}\right), \\
& \left(M_{\pi} a_{\mathrm{LET}, \mathrm{NLO}}^{\left({ }^{3} S_{1}\right)}\right)^{-1}=0.196\left({ }_{-0.013}^{+0.014}\right)\left({ }_{-0.004}^{+0.007}\right), \quad M_{\pi} r_{\text {LET, NLO }}^{\left({ }^{3} S_{1}\right)}=2.44\left({ }_{-0.08}^{+0.08}\right)\left({ }_{-0.17}^{+0.12}\right),
\end{aligned}
$$

where uncertainties in the first brackets correspond to the error bars of the input deuteron binding energy of Eq. (3.1), and for NLO results the second error bars correspond to the variation of the NLO parameter $\delta \beta=0.5$. These results are in contradiction with the values obtained by the NPLQCD Collaboration, given in Eq. (3.3). Specifically, our effective range is a factor of three smaller than the result of NPLQCD.

The effective-range-expansion fit of Ref. [3] may suffer from self-inconsistency issues discussed in Ref. [29]. To illustrate this point we consider the relation between the scattering amplitude and the effective range function given by Eq. (2.5). According to Eq. (2.5), every solution of the equation

$$
F(k)=i k
$$

corresponds to a pole in the scattering amplitude. Graphical solutions of this equation are visualized in Fig. 1 (right), where each intersection of $F\left(k^{2}\right)$ with the $i k$ term (dotted curve) indicates a bound or virtual state. The function $F\left(k^{2}\right)$ corresponding to the ERE fit done in Ref. [3] crosses the $i k$ term twice: the first intersection corresponds to Eq. (3.1) and the second intersection in the vicinity of zero corresponds to a shallow pole in the scattering amplitude. This implies that the deeper bound state (Eq. (3.1)) is outside of the ERE validity range, and the ERE fit is not self-consistent. See Ref. [29] for the extended discussion of this issue.

\subsection{Application of LETs to ${ }^{1} S_{0}$ channel}

In this section we use the LETs to check consistency of the lattice results in the ${ }^{1} S_{0}$ channel. First, we take the NPLQCD's dineutron binding energy in Eq. (3.1) as input for the LETs and predict the ${ }^{1} S_{0}$ phase shifts at LO and NLO. Our results are shown in Fig. 2 as orange (LO) and 

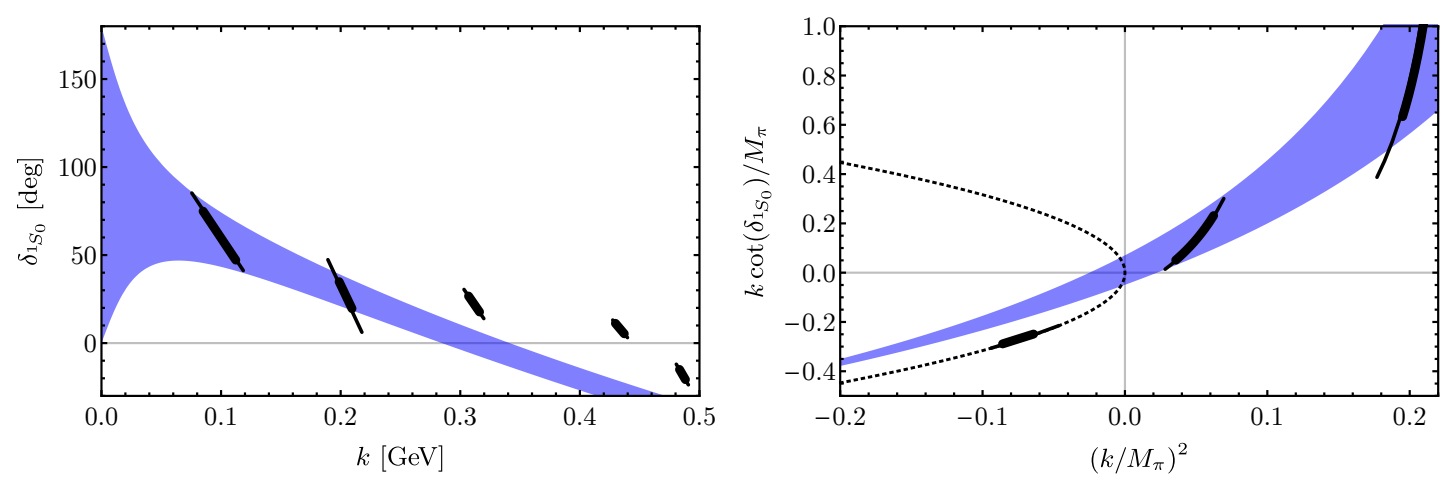

Figure 3: Two-nucleon phase shifts (left panel) and the effective-range function (right panel) in the ${ }^{1} S_{0}$ channel calculated on the lattice at $M_{\pi} \sim 450 \mathrm{MeV}$ [3] in comparison with the predictions based on the LETs at NLO (blue shaded bands) using the scattering length in Eq. (3.8) as input. For remaining notation see Fig. 1.

blue (NLO) bands. Notice, that the shift between LO and NLO LETs results in the ${ }^{1} S_{0}$ channel is larger than in the ${ }^{3} S_{1}$ case because the OPEP strength (and thus the predictive power of LETs) is lower in the ${ }^{1} S_{0}$. For this reason, to estimate NLO LETs theoretical uncertainty in the ${ }^{1} S_{0}$ channel we use an increased range of the NLO parameter corresponding to $\delta \beta=1$. Expanding the phase shift predicted by LETs, we get the scattering length and effective range:

$$
\begin{aligned}
\left(M_{\pi} a_{\mathrm{LET}, \mathrm{LO}}^{\left({ }^{1} S_{0}\right)}\right)^{-1}=0.244\left({ }_{-0.051}^{+0.026}\right), & M_{\pi} r_{\text {LET, LO }}^{\left({ }^{1} S_{0}\right)}=0.90\left({ }_{-0.06}^{+0.14}\right), \\
\left(M_{\pi} a_{\text {LET, NLO }}^{\left({ }^{1} S_{0}\right)}\right)^{-1}=0.175\left({ }_{-0.028}^{+0.013}\right)\left({ }_{-0.008}^{+0.024}\right), & M_{\pi} r_{\text {LET, NLO }}^{\left({ }^{1} S_{0}\right)}=2.86\left({ }_{-0.12}^{+0.27}\right)\left({ }_{-0.74}^{+0.27}\right),
\end{aligned}
$$

where the NLO theoretical uncertainty is calculated assuming $\delta \beta=1$. Comparing ${ }^{1} S_{0}$ LETs predictions with lattice results we obtain similar conclusions as for the ${ }^{3} S_{1}$ channel. Namely, the phase shifts are consistent at larger energies, but disagree at lower ones. Consequently, the LETs results for $a$ and $r$ also disagree with the NPLQCD fit, which has the same self-consistency issues as in the ${ }^{3} S_{1}$ case.

Low-energy theorems can be also used to predict binding energies from low-energy phase shifts data (or, equivalently, from ERE parameters). To illustrate this application, we choose the value of the ${ }^{1} S_{0}$ scattering length in a range which is consistent with the two lowest phase shift data points on the lattice:

$$
\left(M_{\pi} a^{\left({ }^{1} S_{0}\right)}\right)^{-1}=-0.01 \pm 0.06
$$

Using this scattering length as input for LETs, we calculate the phase shifts and the dineutron binding energy. Predicted phase shifts and the corresponding effective range function are shown in Fig. 3. In this case, the dineutron shows up as a shallow bound (or virtual) state with the energy:

$$
B_{n n}<0.5 \mathrm{MeV} \quad B_{n n}^{\text {virtual }}<0.6 \mathrm{MeV}
$$

Note that in this case, there is no deeper bound state. 
A. A. Filin,

\section{Conclusion}

Low-energy theorems can be understood as correlations between scattering observables, which result from the analytic properties of the one-pion exchange potential - the longest-range part of the $\mathrm{NN}$ interaction. We have applied low-energy theorems to $\mathrm{NN}$ scattering at unphysical pion masses to check and augment recent lattice QCD results.

Specifically, we have shown that the binding energies of the deuteron and dineutron obtained by the NPLQCD Collaboration at the pion mass of $M_{\pi}=450 \mathrm{MeV}$ are inconsistent with the lowenergy behavior of the corresponding phase shifts within the quoted uncertainties. This may indicate underestimated systematic uncertainties or issues with the determination of phase shifts or the binding energies on the lattice.

We use the NPLQCD binding energies of the deuteron and dineutron as input for the lowenergy theorems to predict the phase shifts and to extract the scattering length and the effective range in the ${ }^{3} S_{1}$ and ${ }^{1} S_{0}$ channels. The values we obtain are much smaller in magnitude than the corresponding lattice results. If we use the lattice value of the ${ }^{1} S_{0}$ scattering length as input for LETs, then the dineutron is expected to be a shallow bound or virtual state.

We have illustrated how LETs can be used to predict scattering observables for unphysical pion masses and to perform consistency checks of lattice results when several scattering observables are calculated on the lattice.

\section{Acknowledgments}

We would like to thank J. Gegelia for careful reading of the manuscript. This work was supported in part by the DFG (Grant No. TRR110) and the NSFC (Grant No. 11621131001) through the funds provided to the Sino-German CRC 110 "Symmetries and the Emergence of Structure in QCD". Work of V.B. was supported by the Russian Science Foundation (Grant No. 18-12-00226).

\section{References}

[1] S. R. Beane et al. [NPLQCD Collaboration], Phys. Rev. D 85, 054511 (2012), [arXiv:1109.2889 [hep-lat]].

[2] T. Yamazaki, K. i. Ishikawa, Y. Kuramashi and A. Ukawa, Phys. Rev. D 92, no. 1, 014501 (2015), [arXiv:1502.04182 [hep-lat]].

[3] K. Orginos, A. Parreno, M. J. Savage, S. R. Beane, E. Chang and W. Detmold, Phys. Rev. D 92, no. 11, 114512 (2015) [arXiv:1508.07583 [hep-lat]].

[4] T. Doi et al., EPJ Web Conf. 175, 05009 (2018), [arXiv:1711.01952 [hep-lat]].

[5] H. Nemura et al., EPJ Web Conf. 175, 05030 (2018), [arXiv:1711.07003 [hep-lat]].

[6] S. R. Beane et al. [NPLQCD Collaboration], Phys. Rev. D 87, no. 3, 034506 (2013), [arXiv:1206.5219 [hep-lat]].

[7] S. R. Beane et al. [NPLQCD Collaboration], Phys. Rev. C 88, no. 2, 024003 (2013), [arXiv:1301.5790 [hep-lat]]. 
A. A. Filin,

[8] M. Wagman, these proceedings.

[9] E. Berkowitz, T. Kurth, A. Nicholson, B. Joo, E. Rinaldi, M. Strother, P. M. Vranas and A. Walker-Loud, Phys. Lett. B 765, 285 (2017), [arXiv:1508.00886 [hep-lat]].

[10] A. Walker-Loud, these proceedings.

[11] T. Yamazaki, K. i. Ishikawa, Y. Kuramashi and A. Ukawa, Phys. Rev. D 86, 074514 (2012), [arXiv:1207.4277 [hep-lat]].

[12] T. Inoue et al. [HAL QCD Collaboration], Nucl. Phys. A 881, 28 (2012), [arXiv:1112.5926 [hep-lat]].

[13] S. R. Beane, P. F. Bedaque, M. J. Savage and U. van Kolck, Nucl. Phys. A 700, 377 (2002), [nucl-th/0104030].

[14] S. R. Beane and M. J. Savage, Nucl. Phys. A 713, 148 (2003), [hep-ph/0206113].

[15] E. Epelbaum, U.-G. Meißner and W. Glöckle, Nucl. Phys. A 714, 535 (2003), [nucl-th/0207089].

[16] S. R. Beane and M. J. Savage, Nucl. Phys. A 717, 91 (2003), [nucl-th/0208021].

[17] E. Epelbaum, U.-G. Meißner and W. Glöckle, nucl-th/0208040.

[18] J. W. Chen, T. K. Lee, C.-P. Liu and Y. S. Liu, Phys. Rev. C 86, 054001 (2012), [arXiv:1012.0453 [nucl-th]].

[19] J. Soto and J. Tarrus, Phys. Rev. C 85, 044001 (2012), [arXiv:1112.4426 [nucl-th]].

[20] E. Epelbaum and J. Gegelia, PoS CD 12, 090 (2013), [arXiv:1301.6134 [nucl-th]].

[21] J. C. Berengut et al., Phys. Rev. D 87, no. 8, 085018 (2013), [arXiv:1301.1738 [nucl-th]].

[22] V. Baru, E. Epelbaum, A. A. Filin and J. Gegelia, Phys. Rev. C 92, no. 1, 014001 (2015), [arXiv:1504.07852 [nucl-th]].

[23] T. D. Cohen and J. M. Hansen, Phys. Rev. C 59, 13 (1999), [nucl-th/9808038].

[24] T. D. Cohen and J. M. Hansen, Phys. Rev. C 59, 3047 (1999), [nucl-th/9901065].

[25] J. V. Steele and R. J. Furnstahl, Nucl. Phys. A 645, 439 (1999), [nucl-th/9808022].

[26] E. Epelbaum and J. Gegelia, Eur. Phys. J. A 41, 341 (2009), [arXiv:0906.3822 [nucl-th]].

[27] E. Epelbaum and J. Gegelia, PoS CD 09, 077 (2009).

[28] E. Epelbaum, arXiv:1001.3229 [nucl-th].

[29] V. Baru, E. Epelbaum and A. A. Filin, Phys. Rev. C 94, no. 1, 014001 (2016), [arXiv:1604.02551 [nucl-th]].

[30] H. van Haeringen and L. P. Kok, Phys. Rev. A26, 1218 (1982).

[31] V. G. Kadyshevsky, Nucl. Phys. B 6, 125 (1968).

[32] E. Epelbaum and J. Gegelia, Phys. Lett. B 716, 338 (2012), [arXiv:1207.2420 [nucl-th]].

[33] J. M. Blatt and J. D. Jackson, Phys. Rev. 76, 18 (1949).

[34] H. A. Bethe, Phys. Rev. 76, 38 (1949).

[35] J. M. Blatt and L. C. Biedenharn, Rev. Mod. Phys. 24, 258 (1952). 